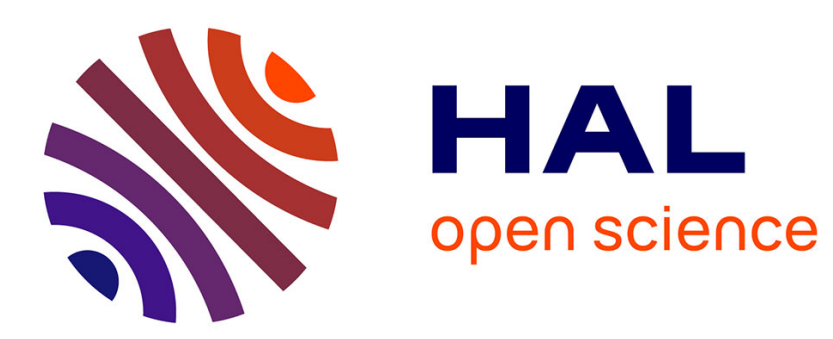

\title{
Maximum feedback linearization with internal stability of 2-DOF underactuated mechanical systems
}

\author{
Divine Maalouf, Claude H. Moog, Yannick Aoustin, Shun-Jie Li
}

\section{To cite this version:}

Divine Maalouf, Claude H. Moog, Yannick Aoustin, Shun-Jie Li. Maximum feedback linearization with internal stability of 2-DOF underactuated mechanical systems. 18th IFAC World Congress, Aug 2011, Milano, Italy. hal-00584325

\section{HAL Id: hal-00584325 \\ https://hal.science/hal-00584325}

Submitted on 8 Apr 2011

HAL is a multi-disciplinary open access archive for the deposit and dissemination of scientific research documents, whether they are published or not. The documents may come from teaching and research institutions in France or abroad, or from public or private research centers.
L'archive ouverte pluridisciplinaire HAL, est destinée au dépôt et à la diffusion de documents scientifiques de niveau recherche, publiés ou non, émanant des établissements d'enseignement et de recherche français ou étrangers, des laboratoires publics ou privés. 


\title{
Maximum feedback linearization with internal stability of 2-DOF underactuated mechanical systems
}

\author{
D. Maalouf* C. H. Moog ${ }^{* *}$ Y. Aoustin ${ }^{* *}$ S. J. Li ${ }^{* *}$ \\ * LIRMM, UMR 5506 C.N.R.S, Université Montpellier 2, 161 rue \\ Ada, 34095 Montpellier Cedex 5, France \\ ** IRCCyN, UMR 6597 C.N.R.S, Ecole Centrale de Nantes, Université \\ de Nantes, BP 92101, 1 rue de la Noë, 44321 Nantes cedex 03, France, \\ (e-mail: Claude.Moog@irccyn.ec-nantes.fr)
}

\begin{abstract}
Maximum feedback linearization with internal stability is considered. A classification of popular mechanical systems is derived, depending on the achievable closed loop system under the constraint of internal stability. It is shown that the angular momentum plays a crucial role for this class of systems. The study includes the special case of a paraglider which consists essentially of two articulated bodies.
\end{abstract}

Keywords: Stability, Equilibrium, Nonlinear control systems, Mechanical systems, Feedback linearization.

\section{INTRODUCTION}

Exact feedback linearization lies in the heart of the success of modern nonlinear control theory for over three decades. Input-output linearization generalizes the socalled computed torque method which has been popularized in robotics. However, some major drawbacks are mentioned from time to time, amongst the existence of singularities, the cancelation of useful nonlinear terms (Ortega et al. (2002)) or the nonobservability of the socalled zero dynamics which may destabilize the closed loop system whenever the system is non minimum phase.

Herein, the problem of exact linearization with internal stability is addressed. The core consists in the design of a dummy output, whenever it exists, such that the underlying system becomes minimum phase. This goal is of major importance by its own as it is a standing assumption for many control schemes including sliding modes or PID feedback...

A general theory for this issue is not available yet. The goal in this paper is to consider a class of underactuated systems whose stabilization is a challenging issue. A general frame is displayed to design a suitable control law for three subclasses of systems according to their structure. One class corresponds to fully linearizable (or flat) systems. It includes the inertia wheel pendulum (Ortega et al. (2002), Beznos et al. (2003)). The second class includes the celebrated Acrobot which has already been worked out by Cambrini et al. (2000) and shown to be linearizable up to order 3 with stable, critically stable or unstable internal dynamics, depending on the choice of output coordinates. The third class is much more challenging and contains systems as the Pendubot (Aoustin et al. (2010)) and a model of paraglider on which a special attention is focused. Such a classification of simple mechanical systems was considered for different purposes in Olfati-Saber (2001).
The paraglider is considered to be equivalent to a double pendulum. The control input is the thrust force applied at the joint between both links. It is proven that the system is linearizable up to order 3 , with a critical internal stability. The main contribution in that respect is to find a suitable output function with relative degree 2 such that the feedback linearization yields asymptotic stability of the full state. The importance of the generalized angular momentum in the stability of the system is argued and the physical interpretation of the functions obtained from the control algorithms is discussed as well.

In Section 2, some standard results are recalled from Marino (1986), Conte et al. (2006) on input-output linearization. The main results are given in Section 3. A general underactuated double pendulum is modeled, so that the Acrobot and the Pendubot become some special cases. Three subclasses of systems are identified and characterized. Section 4 is devoted to the paraglider system for which worked out computations are completed, including some simulation results in Section 5 which make profit of the stability results. Concluding remarks and perspectives are offered in Section 6.

\section{MAXIMUM FEEDBACK LINEARIZATION WITH STABILITY}

Consider a single-input nonlinear system

$$
\Lambda: \quad \dot{x}=f(x)+g(x) u
$$

where the state $x \in \mathbb{R}^{n}$, the control $u \in \mathbb{R}$ and the entries $f, g$ are meromorphic vector fields on $\mathbb{R}^{n}$. Let $\mathcal{C}$ be the infinite set of real indeterminates given by $\mathcal{C}=\left\{x, u^{k} ; k \geq\right.$ $0\}$ and denote by $\mathcal{K}$, a field of meromorphic functions depending on a finite subset of indeterminates of $\mathcal{C}$. We define the time derivative of a function $\varphi \in \mathcal{K}$ as follows 


$$
\dot{\varphi}=\frac{\mathrm{d} \varphi}{\mathrm{d} t}=\frac{\partial \varphi}{\partial x}(f(x)+g(x) u)+\sum_{k \geq 0} \frac{\partial \varphi}{\partial u^{(k)}} u^{(k+1)} .
$$

Let $\mathcal{E}$ denote the vector space spanned over $\mathcal{K}$ by the elements of $\mathrm{d} \mathcal{C}$, namely $\mathcal{E}=\operatorname{span}_{\mathcal{K}}\left\{\mathrm{d} x, \mathrm{~d} u^{(k)} ; k \geq 0\right\}$. Any element of $\mathcal{E}$ is in the form

$$
\omega=a \mathrm{~d} x+\sum_{k \geq 0} b_{k} \mathrm{~d} u^{(k)}
$$

which is called a differential one-form and its time derivative is defined by

$$
\dot{\omega}=(\dot{a} \mathrm{~d} x+a \mathrm{~d} \dot{x})+\sum_{k \geq 0}\left(\dot{b}_{k} \mathrm{~d} u^{(k)}+b_{k} \mathrm{~d} u^{(k+1)}\right) .
$$

The relative degree of a one-form $\omega$ is defined as the least integer $r$ such that $\omega^{(r)} \notin \operatorname{span}_{\mathcal{K}}\{\mathrm{d} x\}$. If it does not exist, we say that $r=\infty$. Similarly, the relative degree of a function $\varphi \in \mathcal{K}$ is defined as the least integer such that $\mathrm{d} \varphi^{(r)} \notin \operatorname{span}_{\mathcal{K}}\{\mathrm{d} x\}$. We have the following result (see Conte et al. (2006))

Proposition 2.1. The function $\varphi \in \mathcal{K}$ and the one-form $\mathrm{d} \varphi$ have the same relative degree.

Introduce a sequence of subspace $\left\{\mathcal{H}_{k}\right\}$ of $\mathcal{E}$ by

$$
\begin{aligned}
& \mathcal{H}_{0}=\operatorname{span}_{\mathcal{K}}\{\mathrm{d} x, \mathrm{~d} u\} \\
& \mathcal{H}_{k+1}=\left\{\omega \in \mathcal{H}_{k} \mid \dot{\omega} \in \mathcal{H}_{k}\right\}, k \geq 1 .
\end{aligned}
$$

Each subspace $\mathcal{H}_{k}$ denotes the set of all one-forms with relative degree at least $k$. Clearly, sequence (2.2) is decreasing, i.e., $\mathcal{E} \supset \mathcal{H}_{0} \supset \mathcal{H}_{1} \supset \mathcal{H}_{2} \cdots$, and we have $\mathcal{H}_{1}=\operatorname{span}_{\mathcal{K}}\{\mathrm{d} x\}$. Note that $\mathcal{H}_{2}=\operatorname{span}_{\mathcal{K}}\{g\}^{\perp}$.

Denote $k^{*}$ the least integer such that $\mathcal{H}_{0} \supset \mathcal{H}_{1} \supset$ $\cdots \mathcal{H}_{k^{*}} \supset \mathcal{H}_{k^{*}+1}=\mathcal{H}_{k^{*}+2}=\cdots \mathcal{H}_{\infty}$. Then system $\Lambda$ is strongly accessible if and only if it satisfies $\mathcal{H}_{\infty}=0$ (see Conte et al. (2006)). In this paper, all the mechanical systems with which we work satisfy $\mathcal{H}_{\infty}=0$.

Let $y=h(x) \in \mathbb{R}$ be an output of $\Lambda$, where $h$ is a meromorphic function, and consider the corresponding SISO (single-input single-output) nonlinear system

$$
\Sigma:\left\{\begin{array}{l}
\dot{x}=f(x)+g(x) u \\
y=h(x) .
\end{array}\right.
$$

Assume that $\Sigma$ has a relative degree $r \leq n$ at some point $x_{0}$, i.e., the relative degree of the output function $y=h(x)$ equal $r$, then locally there exist a regular static state feedback $u=\alpha(x)+\beta(x) v$ and a state transformation $(z, w)=\phi(x)$, where $z=\left(z_{1}, \ldots, z_{r}\right)$, $w=\left(w_{1}, \ldots, w_{n-r}\right)$ and $\phi$ is a diffeomorphism, such that in the $(z, w)$-coordinates, system $\Sigma$ reads, around $x_{0}$,

$$
\begin{aligned}
\dot{z}_{1} & =z_{2} \\
\dot{z}_{2} & =z_{3} \\
& \vdots \\
\dot{z}_{r} & =v \\
\dot{w} & =\eta(z, w) \\
y & =z_{1} .
\end{aligned}
$$

The system description (2.4) is called the normal form for the SISO system $\Sigma$. Consequently, it can be easily seen that $\Sigma$ is fully state linearizable if and only if there exists an output function $y=h(x)$ that has relative degree $n$ at $x_{0}$. Clearly the variables $w$ are unobservable since $z$ do not depend on $w$ at all and the equation $\dot{w}=\eta(z, w)$ represents the "internal dynamics".
Definition 2.2. The zero dynamics of system $\Sigma$, given by (2.3), is defined by the dynamics

$$
\dot{w}=\eta(0, w)
$$

which are the internal dynamics consistent with the constraint that $y(t) \equiv 0$.

In this paper, we will study the maximal feedback linearization with internal stability of the 2 DOF underactuated mechanical system. More precisely, given a single-input nonlinear system $\Lambda: \quad \dot{x}=f(x)+g(x) u$, we want to find an output $y=h(x) \in \mathbb{R}$ with the maximal relative degree $r$ and having the following property: there exist a regular static state feedback $u=\alpha(x)+\beta(x) v$ and a state transformation $(z, w)=\phi(x)$, where $z=\left(z_{1}, \ldots, z_{r}\right)$, $w=\left(w_{1}, \ldots, w_{n-r}\right)$ and $\phi$ is a diffeomorphism, such that in the $(z, w)$-coordinates the corresponding SISO system $\Sigma$ can be transformed into the normal form (2.4) and simultaneously the zero dynamics is (asymptotically) stable.

\section{A GENERAL CLASS OF 2-DOF SYSTEMS}

Consider the double link pendulum shown in Figure 1. It is assumed to be attached to a fixed pivot point $O_{1}$

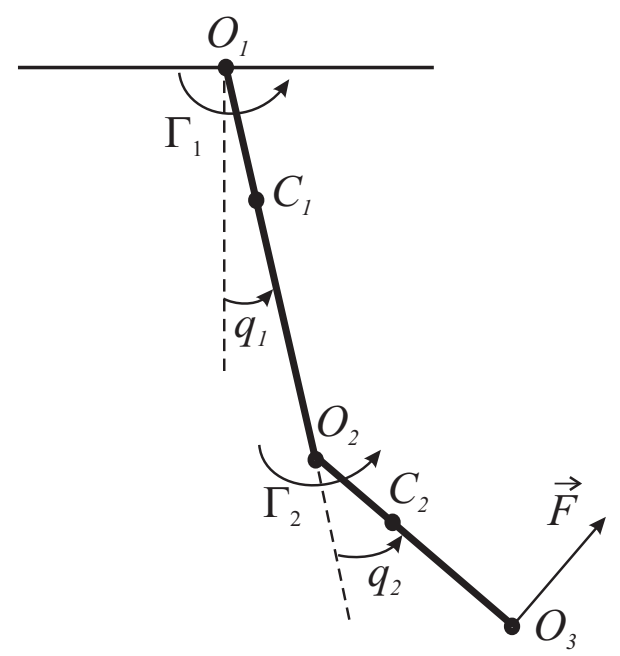

Fig. 1. Scheme of a double link pendulum.

creating angle $q_{1}$. Angle $q_{2}$ is the joint angle between links. Let $C_{1}$ and $C_{2}$ be the centers of mass of the first and second link respectively. The center of mass $C_{1}$ is located on line $O_{1} O_{2}$. Let the following lengths be $O_{1} O_{2}=l_{1}$, $\mathrm{O}_{2} \mathrm{O}_{3}=l_{2}, O_{1} C_{1}=r_{1}$ and $O_{2} C_{2}=r_{2}$. Let $m_{1}$ and $m_{2}$ be the masses of the first and second links. The moment of inertia of the first link about joint $O_{1}$ is denoted $I_{1}$, the moment of inertia of the second link about joint $O_{2}$ is denoted $I_{2}$. At the tip of the second link $\mathrm{O}_{2}$ an external force $\vec{F}=\left[F_{x}, F_{y}\right]^{\mathrm{t}}$ is applied.

The expressions for the kinetic energy $T$ and the potential energy $\Pi$ of the two-link pendulum are well known:

$$
\begin{gathered}
2 T=a_{11} \dot{q}_{1}^{2}+2 a_{21} \cos q_{2} \dot{q}_{1}^{2}+a_{22} \dot{q}_{1}^{2}+ \\
\left(a_{22}+a_{21} \cos q_{2}\right) \dot{q}_{1} \dot{q}_{2}+a_{22} \dot{q}_{2}^{2} \\
\Pi=-b_{1} \cos q_{1}-b_{2} \cos \left(q_{1}+q_{2}\right),
\end{gathered}
$$

with $a_{11}=I_{1}+m_{2} l_{1}^{2}, a_{21}=m_{2} r_{2} l_{1}, a_{22}=I_{2}, b_{1}=\left(m_{1} r_{1}+\right.$ $\left.m_{2} l_{1}\right) g, b_{2}=m_{2} r_{2} g$ where $g$ is the gravity acceleration. 
Lagrangian $L=T-\Pi$ yields the following well known matrix equation of motion:

$$
D(q) \ddot{q}+C(q, \dot{q}) \dot{q}+G(q)=J^{\mathrm{t}} F
$$

where

$$
\begin{gathered}
q=\left[q_{1}, q_{2}\right]^{\mathrm{t}} \\
D(q)=\left[\begin{array}{cc}
a_{11}+a_{22}+2 a_{21} \cos q_{2} & a_{22}+a_{21} \cos q_{2} \\
a_{22}+a_{21} \cos q_{2} & a_{22}
\end{array}\right], \\
C(q, \dot{q})=\left[\begin{array}{cc}
-a_{21} \dot{q}_{2} \sin q_{2} & -a_{21}\left(\dot{q}_{1}+\dot{q}_{2}\right) \sin q_{2} \\
a_{21} \dot{q}_{1} \sin q_{2} & 0
\end{array}\right], \\
G(q)=\left[\begin{array}{c}
b_{1} \sin q_{1}+b_{2} \sin \left(q_{1}+q_{2}\right) \\
b_{2} \sin \left(q_{1}+q_{2}\right)
\end{array}\right] .
\end{gathered}
$$

The matrix $J$ is the Jacobian of the forward kinematics. The arbitrarily directed force makes a torque at each joint such that:

$$
\begin{aligned}
& \Gamma=\left[\begin{array}{l}
\Gamma_{1} \\
\Gamma_{2}
\end{array}\right]=J^{\mathrm{t}} F= \\
& {\left[\begin{array}{cc}
l_{2} \cos \left(q_{1}+q_{2}\right)+l_{1} \cos q_{1} & l_{2} \sin \left(q_{1}+q_{2}\right)+l_{1} \sin q_{1} \\
l_{2} \cos \left(q_{1}+q_{2}\right) & l_{2} \sin \left(q_{1}+q_{2}\right)
\end{array}\right]\left[\begin{array}{c}
F_{x} \\
F_{y}
\end{array}\right] .}
\end{aligned}
$$

A double link pendulum can define a family of underactuated mechanical systems categorized according to the orientation of an external force $\vec{F}$ acting on this pendulum. Consider for example the end force $\vec{F}$. When $\vec{F}$ is parallel to the segment $\mathrm{O}_{2} \mathrm{O}_{3}$, we are in the case of the Pendubot and when it is directed towards $\mathrm{O}_{1} \mathrm{O}_{3}$, we are in the case of the Acrobot. See Figure 2, hence, these systems are classified according to which joint is actuated: $\Gamma=\left[\Gamma_{1}, 0\right]^{\mathrm{t}}$ for the Pendubot, $\Gamma=\left[0, \Gamma_{2}\right]^{\mathrm{t}}$ for the Acrobot. In nominal regime the paraglider is also represented using
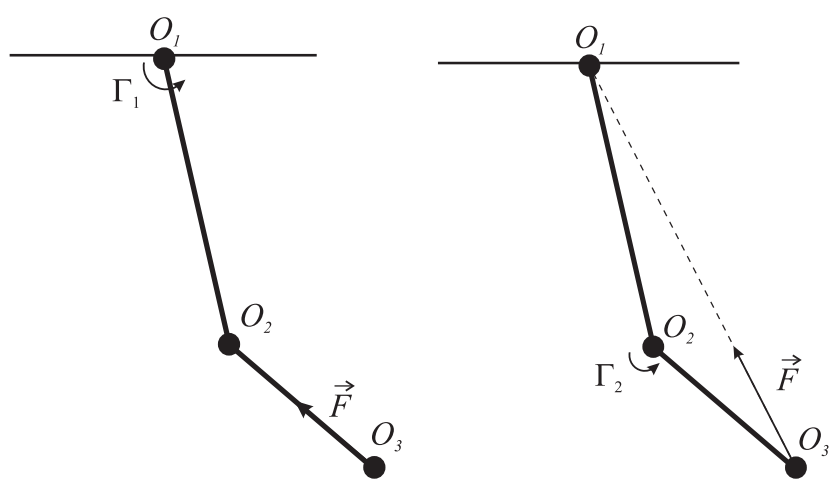

Fig. 2. Left. Pendubot. Right. Acrobot.

the previous double link mechanism, see Figure 3. The first link regroups the canopy and the risers. The second link represents the gondola. At the connection point $\mathrm{O}_{2}$ between both links, the external force $\vec{F}=\left[F_{x}, 0\right]^{\mathrm{t}}$, assumed to be horizontal, represents the thrust of the motor, see Zaitsev and Formal'skii (2008). This force makes a moment $\Gamma_{1}=F_{x} l_{1} \cos q_{1}$ at point $O_{1}, \Gamma_{2}=0$ at point $O_{2}$.

\subsection{Class 0: Inertia wheel pendulum}

An underactuated two-bodies mechanical system is of class 0 if it has a relative degree four with respect to the input. For example consider a pendulum with a balanced flywheel at the end, as shown in Figure 4. The joint variable of the pendulum and the angle of the flywheel rotation are denoted as $q_{1}$ and $q_{2}$ respectively. Let $C$ be the center of

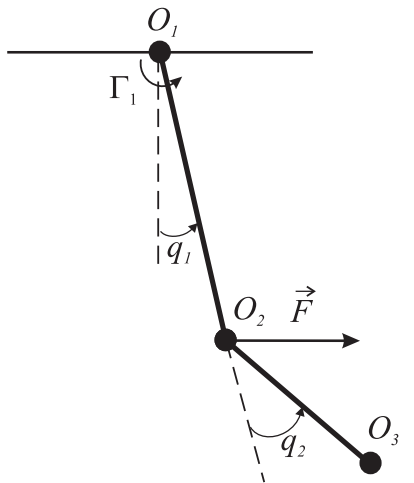

Fig. 3. Scheme of the paraglider.

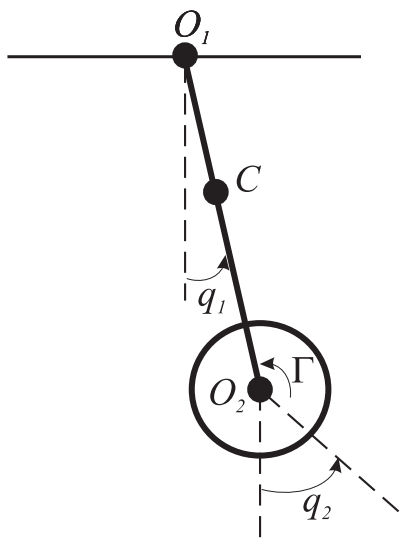

Fig. 4. Scheme of the inertia wheel pendulum.

mass of the link, located on line $\mathrm{O}_{1} \mathrm{O}_{2}$. Let the following lengths be $O_{1} O_{2}=l$ and $O_{1} C=r$. Let $m_{1}$ and $m_{2}$ be the masses of the link and the flywheel. The moment of inertia of the link about joint $O_{1}$ is denoted by $I_{1}$. The inertia moment around the center of mass of the flywheel is denoted by $I_{2}$. Let $m_{1}$ and $m_{2}$ be the masses of the link and the flywheel respectively. The equations of the pendulum motion with the flywheel can be written as (Aoustin et al. (2006))

$$
\frac{\mathrm{d}}{\mathrm{d} t}\left[\begin{array}{c}
q_{1} \\
\dot{q}_{1} \\
q_{2} \\
\dot{q}_{2}
\end{array}\right]=\left[\begin{array}{c}
\dot{q}_{1} \\
-\frac{b}{a_{11}} \sin q_{1} \\
\dot{q}_{2} \\
0
\end{array}\right]+\left[\begin{array}{c}
0 \\
-\frac{1}{a_{11}} \\
0 \\
\frac{1}{a_{22}}
\end{array}\right] \Gamma
$$

Here $a_{11}=I_{1}+m_{2} l^{2}, a_{22}=I_{2}, b=\left(m_{1} r+m_{2} l\right) g$, where $g$ is the gravity acceleration. From (3.2), one computes the subspace $\mathcal{H}_{2}$ of $\mathcal{E}$, which consists of all one-forms that need to be differentiated at least twice to depend explicitly on $\mathrm{d} u$ :

$$
\mathcal{H}_{2}=\operatorname{span}_{\mathcal{K}}\left\{\mathrm{d} q_{1}, \mathrm{~d} q_{2}, \mathrm{~d}\left[a_{11} \dot{q}_{1}+a_{22} \dot{q}_{2}\right]\right\}
$$

We can deduce the subspaces $\mathcal{H}_{3}$ and $\mathcal{H}_{4}$ of $\mathcal{E}$, consisting of all one-forms needed to be differentiated three and four times respectively before depending explicitly on the input.

$$
\begin{gathered}
\mathcal{H}_{3}=\operatorname{span}_{\mathcal{K}}\left\{\mathrm{d}\left[a_{11} q_{1}+a_{22} q_{2}\right], \mathrm{d}\left[a_{11} \dot{q}_{1}+a_{22} \dot{q}_{2}\right]\right\}, \\
\mathcal{H}_{4}=\operatorname{span}_{\mathcal{K}}\left\{\mathrm{d}\left[a_{11} q_{1}+a_{22} q_{2}\right]\right\} .
\end{gathered}
$$


The inertia wheel pendulum is a differentially flat system since the following output $y=a_{11} q_{1}+a_{22} q_{2}$ has a degree 4. We have:

$$
\begin{aligned}
& \dot{y}=a_{11} \dot{q}_{1}+a_{22} \dot{q}_{2} \\
& \ddot{y}=-b \sin q_{1} \\
& y^{(3)}=-b \dot{q}_{1} \cos q_{1} \\
& y^{(4)}=b \dot{q}_{1}^{2} \sin q_{1}-b\left[\frac{b}{a_{11}} \sin q_{1}-\frac{\Gamma}{a_{11}}\right]
\end{aligned}
$$

\subsection{Class 1: Acrobot}

The acrobot has only one cyclic variable, $q_{1}$, which is unactuated. In Cambrini et al. (2000) and Grizzle et al. (2005) it is proven that the underactuated two-bodymechanical system is of class 1 if and only if $\mathcal{H}_{3}$ is fully integrable. Moreover, the maximal linearization of order 3 can be performed with internal stability. In terms of full integrability of $\mathcal{H}_{3}$, the Acrobot belongs to class 1 systems with

$$
\mathcal{H}_{3}=\operatorname{span}_{\mathcal{K}}\left\{\mathrm{d} \sigma, \mathrm{d} p_{1}\right\}
$$

where $\sigma$ is the angular momentum with respect to the suspension joint $O_{1}$ of the double-link pendulum and we have

$$
\begin{aligned}
& \sigma=\frac{\partial T}{\partial \dot{q}_{1}}= \\
& \left(a_{11}+a_{22}+2 a_{21} \cos q_{2}\right) \dot{q}_{1}+\left(a_{22}+a_{21} \cos q_{2}\right) \dot{q}_{2},
\end{aligned}
$$

and

$$
\begin{aligned}
\mathrm{d} p_{1} & =\frac{\mathrm{d} \sigma}{a_{11}+a_{22}+2 a_{21} \cos q_{2}} \\
& =\mathrm{d} q_{1}+\frac{a_{22}+a_{21} \cos q_{2}}{a_{11}+a_{22}+2 a_{21} \cos q_{2}} \mathrm{~d} q_{2},
\end{aligned}
$$

A direct computation gives

$$
\begin{aligned}
& p_{1}=q_{1}+\frac{q_{2}}{2}+ \\
& A \arctan \left(\sqrt{\frac{a_{11}+a_{22}-2 a_{21}}{a_{11}+a_{22}+2 a_{21}}} \tan \frac{q_{2}}{2}\right)+K
\end{aligned}
$$

with $A=\frac{a_{22}-a_{11}}{\sqrt{\left(a_{11}+a_{22}\right)^{2}-4 a_{21}^{2}}}$ and $K$ is a constant.

For the output function

$$
\begin{aligned}
y & =K_{1} p_{1}(q)+K_{2} \sigma(q, \dot{q}) \\
& =K_{1} p_{1}(q)+K_{2}\left(a_{11}+a_{22}+2 a_{21} \cos q_{2}\right) p_{1}(q, \dot{q}),
\end{aligned}
$$

it can be shown that the resulting zero dynamics is asymptotically stable, whenever $K_{1} / K_{2}$ is positive.

\subsection{Class 2: Pendubot, Paraglider}

For systems of class 2 such as the Pendubot and the Paraglider, $\mathcal{H}_{3}$ is also of dimension 2. However, $\mathcal{H}_{3}$ is not fully integrable any more but contains one exact differential 1-form. In this case only one independant function has a relative degree, which is equal to 3 . The attached zero dynamics is unique and there is no freedom to design any alternative output functions as it was the case for the class 1 system. To analyze the situation from a physical point of view, let us consider again the angular momentum (3.7). For the Pendubot the actuator is located at the pivot point and makes an external moment. For the Paraglider, the force applied at the connection point between the links represents the thrust due to the propeller and is considered external. Then this force has to be considered as external. The time derivative of (3.7) is equal to the moment of the external forces applied on the system. Therefore, for both systems, the angular momentum has a relative degree of 1 since the input appears at the first differentiation. They belong to the family, called class 2 systems. Let us now detail $\mathcal{H}_{i}, i=1,2$ for the paraglider case. Writing (3.1) in state space form and choosing the angular variables of the link and their time derivatives as state variables we get a compact matrix equation:

$$
\begin{aligned}
\frac{\mathrm{d}}{\mathrm{d} t}\left[\begin{array}{c}
q_{1} \\
\dot{q}_{1} \\
q_{2} \\
\dot{q}_{2}
\end{array}\right]=\left[\begin{array}{c}
\dot{q}_{1} \\
f_{1}(q, \dot{q}) \\
\dot{q}_{2} \\
f_{2}(q, \dot{q})
\end{array}\right]+ \\
\frac{1}{a_{11} a_{22}-a_{21}^{2}}\left[\begin{array}{c}
0 \\
a_{22} r_{1} \cos q_{1} \\
0 \\
-\left(a_{22}+a_{21} \cos q_{2}\right) r_{1} \cos q_{1}
\end{array}\right] F_{x}
\end{aligned}
$$

where $f_{i}(q, \dot{q}), i=1,2$ are independent from the input. To find the order of maximal linearization of the system we compute the $\mathcal{H}_{k}$ spaces. A straightforward calculation shows the $\mathcal{H}_{2}$ space, the set of all the one-form with the relative degree at least 2 , can be expressed as

$$
\mathcal{H}_{2}=\operatorname{span}_{\mathcal{K}}\{g\}^{\perp}=\left\{\mathrm{d} q_{1}, \mathrm{~d} q_{2}, \mathrm{~d} \phi\right\}
$$

Three independent functions of relative degree 2 are therefore given by the angular variables $q_{1}$ and $q_{2}$ and:

$$
\phi=\left(a_{22}+a_{21} \cos q_{2}\right) \dot{q}_{1}+a_{22} \dot{q}_{2}
$$

Any vector $\omega$ of $\mathcal{H}_{2}$ can be written as

$$
\omega=a_{\omega} \mathrm{d} q_{1}+b_{\omega} \mathrm{d} q_{2}+c_{\omega} \mathrm{d} \phi
$$

with $a_{\omega}, b_{\omega}$ and $c_{\omega}$ being arbitrary functions. $\mathcal{H}_{3}$ space is given by:

$$
\mathcal{H}_{3}=\left\{\omega \in \mathcal{H}_{2} \mid \dot{\omega} \in \mathcal{H}_{2}\right\} .
$$

with

$$
\dot{\omega}=\dot{a}_{\omega} \mathrm{d} q_{1}+\dot{b}_{\omega} \mathrm{d} q_{2}+\dot{c}_{\omega} \mathrm{d} \phi+a_{\omega} \mathrm{d} \dot{q}_{1}+b_{\omega} \mathrm{d} \dot{q}_{2}+c_{\omega} \mathrm{d} \dot{\phi}
$$

All the terms in $\mathrm{d} q_{1}, \mathrm{~d} q_{2}$ and $\mathrm{d} \phi$ belong to $\mathcal{H}_{2}$ space. Then for $\dot{\omega}$ we have to cancel out the terms in $\mathrm{d} \dot{q}_{1}, \mathrm{~d} \dot{q}_{2}$. After some calculations, two solutions are possible.

The first solution is:

$$
\omega_{1}=\left(a_{22}+a_{21} \cos q_{2}\right) \mathrm{d} \dot{q}_{1}+a_{22} \mathrm{~d} \dot{q}_{2}
$$

By using the integrating factor $\lambda=\frac{1}{a_{22}+a_{21} \cos q_{2}}$ we get the following exact differential form:

$$
\mathrm{d} p_{2}=\lambda \omega_{1}=\mathrm{d} q_{1}+\frac{a_{22}}{a_{22}+a_{21} \cos q_{2}} \mathrm{~d} q_{2}
$$

A direct computation leads to the expression of $p_{2}$

$$
\begin{aligned}
& p_{2}=q_{1}+ \\
& \frac{2 a_{22}}{\sqrt{a_{22}^{2}-a_{21}^{2}}} \arctan \left(\frac{a_{22}-a_{21}}{\sqrt{a_{22}^{2}-a_{21}^{2}}} \tan \frac{q_{2}}{2}\right)+K
\end{aligned}
$$

wehre $K$ is a constant.

The second solution is:

$$
\begin{aligned}
\omega_{2}= & \left(\frac{-a_{21} \sin q_{1} \dot{q}_{1}\left(a_{22}+a_{21} \cos q_{2}\right)}{a_{22}}+\right. \\
& \left.2 a_{21} \sin q_{2} \dot{q}_{1}+a_{21} \dot{q}_{2}\right) \mathrm{d} q_{1}+\mathrm{d} \phi .
\end{aligned}
$$


Thus $\mathcal{H}_{3}$ space is given by:

$$
\mathcal{H}_{3}=\operatorname{span}_{\mathcal{K}}\left\{\omega_{2}, \mathrm{~d} p_{2}\right\} \text {. }
$$

It is easy to check, by Frobenius theorem, that $\mathcal{H}_{3}$ is not integrable.

\section{CONTROL OF THE PARAGLIDER}

The parameter values for the paraglider, modeled by a double-link pendulum, are: $I_{1}=24.4 \mathrm{~kg} \cdot \mathrm{m}^{2}, m_{1}=10 \mathrm{~kg}$, $l_{1}=7 \mathrm{~m}$, and $r_{1}=1 \mathrm{~m}$ for the canopy and $I_{2}=25 \mathrm{~kg} \cdot \mathrm{m}^{2}$, $m_{2}=100 \mathrm{~kg}, l_{2}=1.7 \mathrm{~m}$ and $r_{2}=1 \mathrm{~m}$ for the gondola. The considered constant for the gravity effect is $g=9.81 \mathrm{~m} / \mathrm{s}^{2}$. The aim of the control in nominal regime of the paraglider is to stabilize the pendulum at a fixed position using one input which is the thrust of the propeller. The chosen output function $y$ is made out of the combination of all available functions present in $H_{2}$ space, i.e.,

$$
y=\alpha_{1} \phi+\alpha_{2} q_{1}+\alpha_{3} q_{2}+\alpha_{4}
$$

Let us compute the feedback control force $F_{x}$ by solving the following equation:

$$
\ddot{y}+2 \dot{y}+y=0
$$

From (4.1), equation (4.2) becomes

$$
\alpha_{1} \ddot{\phi}+\alpha_{2} \ddot{q}_{1}+2\left(\alpha_{1} \dot{\phi}+\alpha_{2} \dot{q}_{1}\right)+\alpha_{1} \phi+\alpha_{2} q_{1}+\alpha_{3} q_{2}+\alpha_{4}=0
$$

By using the dynamic model (3.1) and the compact state form (3.10) we can write

$$
\begin{aligned}
& \ddot{\phi}=B_{\phi}+C_{\phi} F_{x} \\
& \ddot{q}_{1}=B_{q_{1}}+C_{q_{1}} F_{x} \\
& \ddot{q}_{2}=B_{q_{2}}+C_{q_{2}} F_{x}
\end{aligned}
$$

where $B_{\phi}, C_{\phi}, B_{q_{1}}, C_{q_{1}}, B_{q_{2}}$ and $C_{q_{2}}$ are functions used to separate the terms that depend on the input from the ones that do not. Finally the feedback control force $F_{x}$ can be written such as:

$$
\begin{aligned}
F_{x}= & \frac{-2\left(\alpha_{1} \dot{\phi}+\alpha_{2} \dot{q}_{1}+\alpha_{3} \dot{q}_{2}\right)}{\alpha_{1} C_{\phi}+\alpha_{2} C_{q_{1}}+\alpha_{3} C_{q_{2}}}- \\
& \frac{\alpha_{1} \phi+\alpha_{2} q_{1}+\alpha_{3} q_{2}+\alpha_{1} B_{\phi}+\alpha_{2} B_{q_{1}}+\alpha_{3} B_{q_{2}}}{\alpha_{1} C_{\phi}+\alpha_{2} C_{q_{1}}+\alpha_{3} C_{q_{2}}}
\end{aligned}
$$

In nominal regime the equilibrium point is defined as

$$
q_{1 e}=-q_{2 e}=-\frac{\alpha_{4}}{\left|\alpha_{2}\right|+\left|\alpha_{3}\right|}
$$

with the numerical data $\dot{q}_{1 e}=\dot{q}_{2 e}=0$ and $q_{1 e}=-q_{2 e}=0.1$. With the proposed family of output variables $y(4.1)$, we choose the following numerical values of the coefficients $\alpha_{1}, \alpha_{2}, \alpha_{3}$ and $\alpha_{4}$ are:

$$
\begin{gathered}
\alpha_{1}=0.005 \mathrm{~s} /\left(\mathrm{rd} . \mathrm{Kg} . \mathrm{m}^{2}\right), \quad \alpha_{2}=1 \mathrm{~s} / \mathrm{rd}, \\
\alpha_{3}=-1 \mathrm{~s} / \mathrm{rd}, \quad \alpha_{4}=-0.2 \mathrm{rd} / \mathrm{s}
\end{gathered}
$$

These numerical values yields to an equilibrium point or in another word a nominal regime $q_{1}=0.1 \mathrm{rd}$ and $q_{2}=-0.1 r d$. There are numerous parameter sets, which yields to other nominal regimes. The output (4.1) have been chosen such that the nonlinear feedback control (4.5), ensures (4.2) and furthermore the stability of the internal dynamics. To check this stability, we can consider the closed system (3.10) with output $y$ (4.1) governed by (4.2) and the control law. Its linearization about the equilibrium point $q_{e}$ results in the linear system: Its linearization about the equilibrium point $q_{e}$ results in the linear system:

$$
\dot{x}=A x
$$

with $x\left[\begin{array}{lll}q_{1}-q_{1 e} & \dot{q}_{1} & q_{2}-q_{2 e} \\ \dot{q}_{2}\end{array}\right]^{\mathrm{t}}$ and

$$
A=\left[\begin{array}{cccc}
0 & 1 & 0 & 0 \\
\left.\frac{\partial \ddot{q}_{1}}{\partial q_{1}}\right|_{q_{e}} & \left.\frac{\partial \ddot{q}_{1}}{\partial \dot{q}_{1}}\right|_{q_{e}} & \left.\frac{\partial \ddot{q}_{1}}{\partial q_{2}}\right|_{q_{e}} & \left.\frac{\partial \ddot{q}_{1}}{\partial \dot{q}_{2}}\right|_{q_{e}} \\
0 & 0 & 0 & 1 \\
\left.\frac{\partial \ddot{q}_{2}}{\partial q_{1}}\right|_{q_{e}} & \left.\frac{\partial \ddot{q}_{2}}{\partial \dot{q}_{1}}\right|_{q_{e}} & \left.\frac{\partial \ddot{q}_{2}}{\partial q_{2}}\right|_{q_{e}} & \left.\frac{\partial \ddot{q}_{2}}{\partial \dot{q}_{2}}\right|_{q_{e}}
\end{array}\right]
$$

The eigenvalues of $A$ obtained are:

$$
\lambda_{1}=\lambda_{2}=-1, \quad \lambda_{3}=-2.889, \quad \lambda_{4}=-0.71679
$$

These eigenvalues are strictly in the left-half complex plane, the equilibrium point $q_{e}$ is locally asymptotically stable for the nonlinear system (3.10)-(4.5)-(4.1) see for example (Khalil (2000)). In conclusion the chosen output function $y$ (4.1) does not have any remarkable singularity problem and most importantly it yields a stable zero dynamics. Note that the parameter values of the paraglider have an important influence on the stability properties. The coefficient values have to be recomputed for whenever different parameters are considered for the paraglider.

\section{SIMULATION RESULTS}

The graphs below show the profiles of the generalized coordinates $q_{1}$ and $q_{2}$, their time derivative $\dot{q}_{1}$ and $\dot{q}_{2}$, the chosen output function $y$ (4.1) with (4.7) and the horizontal thrust $F_{x}$ due to the propeller. The initial conditions are:

$$
q_{1}=0.0 \mathrm{rd}, \dot{q}_{1}=0.05 \mathrm{rd} / \mathrm{s} q_{2}=0.0 \mathrm{rd}, \dot{q}_{2}=0.05 \mathrm{rd} / \mathrm{s}
$$

Figure 5 shows, the thrust of the propeller reaching a constant value, which is close to $100 \mathrm{~N} . \mathrm{m}$. This force $F_{x}$ ensures the convergence of the output variable to $y$, Figure 6. In Figures 7 and 8 , we can see that the zero dynamics of the Paraglider are stable because the generalized coordinates $q_{1}$ and $q_{2}$ converge to constant values and their time derivatives $\dot{q}_{1}$ and $\dot{q}_{2}$ converge to zero. Several sets of initial conditions have been tested, which proved that the basin of attraction of the closed loop system is significantly large.

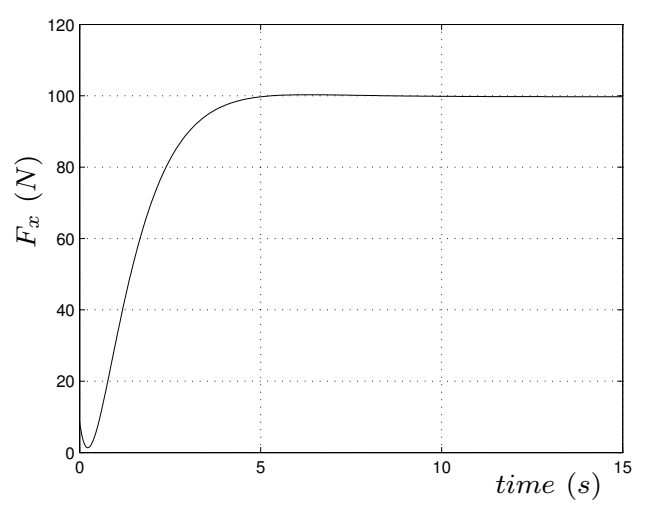

Fig. 5. Profile of the control thrust force $F_{x}$.

\section{CONCLUSION}

It has been possible to state and to solve the maximal linearization problem with internal stability for a general class of mechanical systems. The problem rose from 


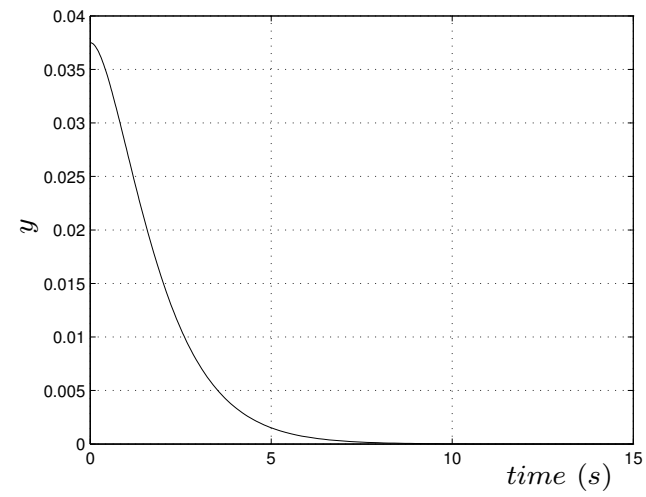

Fig. 6. Profile of the chosen output function $y$.
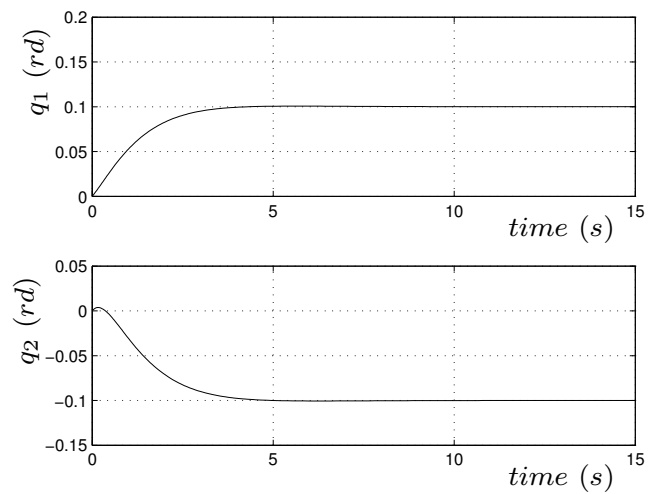

Fig. 7. Profiles of the generalized coordinates of the Paraglider $q_{1}$ and $q_{2}$ radians .
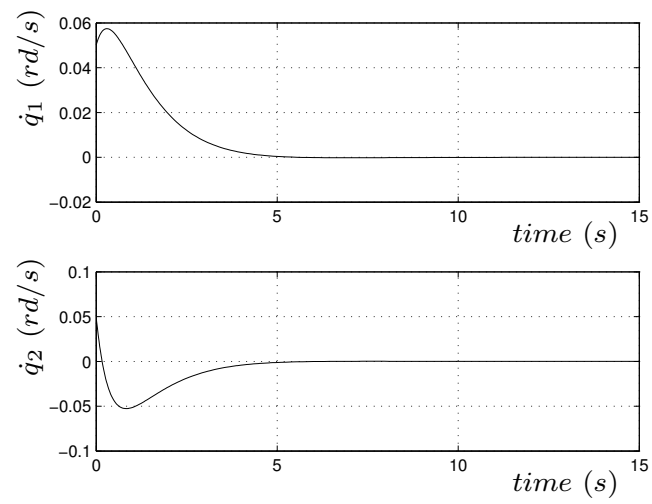

Fig. 8. Profiles of the angular velocities of both links of the Paraglider $\dot{q}_{1}$ and $\dot{q}_{2}$ radians per second.

the control of walking robots for over one decade and such technological domains strongly motivate these studies which deserve an abstract development. Obviously, the problem for the most general nonlinear systems remains difficult to solve as it depends on both on the structure of the system and on the analysis of trajectories. Surprisingly, it was possible to solve the problem for the class of mechanical systems under interest thanks to the generalized angular momentum.

\section{ACKNOWLEDGEMENT}

This work has been accomplished while the first author was in IRCCyN.

\section{REFERENCES}

Y. Aoustin, A. Formalskii, and Y. Martynenko. Stabilization of unstable equilibrium postures of a two-link pendulum using a flywheel. Journal of Computer and Systems Sciences International, 45(2):204-211, 2006.

Y. Aoustin, A. Formalskii, and Y. Martynenko. Pendubot: Combining of energy and intuitive approaches to swing up, stabilization in erected pose. Multibody System Dynamics, Kluwer Academic Publishers, to appear, 2010.

A. V. Beznos, A.A. Grishin, A.V. Lensky, D.E. Okhotsimsky, and A.M. Formal'sky. A pendulum controlled by a flywheel. Doklady Mathematics, 392(6):743-749, 2003.

L. Cambrini, C. Chevallereau, C. H. Moog, and R. Stojic. Stable trajectory tracking for biped robots. In In IEEE Press, editor, Proceedings of the 39th IEEE Conference on Decision and Control, pages 4815-4820, Sydney, Australie, 2000.

G. Conte, C. M. Moog, and A. M. Perdon. Algebraic Methods for Nonlinear Control Systems. 2nd Edition. Springer-Verlag, London, England, 2006.

J.W. Grizzle, C. H. Moog, and C. Chevallereau. Nonlinear control of mechanical systems with an unactuated cyclic variable. IEEE Transactions on Automatic Control, 50 (5):559-576, 2005.

H. Khalil. Nonlinear Systems. Prentice Hall, 2000.

R. Marino. On the largest feedback linearizable subsystem. Systems \& Control Letters, 6(5):345-351, 1986.

R. Olfati-Saber. Nonlinear Control of underactuated mechanical systems with application to robotics and aerospace vehicles. Phd thesis, Massachusetts Institute of Technology, 2001.

R. Ortega, M. W. Spong, F. Gomez-Estern, and G. Blankenstein. Stabilization of a class of underactuated mechanical systems via interconnection and damping assignment. IEEE Trans. on Automatic Control, 47 (8):1218-1233, 2002.

P. V. Zaitsev and A. M. Formal'skii. Paraglider: Mathematical model and control. Doklady Mathematics, 77 (3):1-4, 2008. 